\title{
Using Acoustic Sensor Technologies to Create a More Terrain Capable Unmanned Ground Vehicle
}

\author{
Siddharth Odedra ${ }^{1}$, Stephen D. Prior ${ }^{1}$, Mehmet Karamanoglu ${ }^{1}$, \\ Mehmet Ali Erbil ${ }^{1}$, and Siu-Tsen Shen ${ }^{2}$ \\ ${ }^{1}$ Department of Product Design and Engineering, \\ School of Engineering and Information Sciences, Middlesex University, \\ London N14 4YZ, United Kingdom \\ ${ }^{2}$ Department of Multimedia Design, National Formosa University, \\ 64 Wen-Hua Rd, Hu-Wei 63208, Taiwan \\ s.odedra@mdx.ac.uk
}

\begin{abstract}
Unmanned Ground Vehicle's (UGV) have to cope with the most complex range of dynamic and variable obstacles and therefore need to be highly intelligent in order to cope with navigating in such a cluttered environment. When traversing over different terrains (whether it is a UGV or a commercial manned vehicle) different drive styles and configuration settings need to be selected in order to travel successfully over each terrain type. These settings are usually selected by a human operator in manned systems on what they assume the ground conditions to be, but how can an autonomous UGV 'sense' these changes in terrain or ground conditions? This paper will investigate noncontact acoustic sensor technologies and how they can be used to detect different terrain types by listening to the interaction between the wheel and the terrain. The results can then be used to create a terrain classification list for the system so in future missions it can use the sensor technology to identify the terrain type it is trying to traverse, which creating a more autonomous and terrain capable vehicle. The technology would also benefit commercial driver assistive technologies.
\end{abstract}

Keywords: Unmanned Ground Vehicles, Terrain Sensing, Situational Awareness, Tyre Noise.

\section{Introduction}

Unmanned systems are being used for many applications in nearly every industry where humans are either unwilling or unable to operate in. They operate in many different environments such as on the ground, in the air, under the sea and even out in space. Each of these environments have a range of conditions and obstacles which make it difficult for the unmanned system to operate in, for example wind speed is a key issue for the Unmanned Aerial Vehicle (UAV), as is keeping electronic components from getting wet for the Unmanned Underwater Vehicle (UUV); however because of the number of variables on the ground, the Unmanned Ground Vehicle 
(UGV) has the hardest job in terms of navigating in its environment. Ground conditions are the most difficult to operate in because they usually include dynamic and variable obstacles over a range of different terrain types and systems have to usually operate in unknown, unstructured environments which include a large number of unpredictable variables, making the seemingly simple task of traversing very hard.

Most vehicles that travel on the ground whether manned or unmanned are generally designed to drive over a flat structured road, however when the road conditions change with bad weather or the vehicle is required to go off-road, then the vehicle needs to be capable of coping with a larger range of conditions. To start with the vehicle has to have a very high degree of mobility (such as $4 \times 4$ or tracked vehicles), but in the case of an autonomous system that doesn't have the decision making of a human operator then the vehicle must also have some intelligence in order to help understand the conditions of the local environment to inform an otherwise oblivious system of its situation, so that it can then make decisions on what to do and where to go next in order to complete the mission without becoming stuck.

\section{Vehicle Assistive Systems}

Nowadays even commercial vehicles are equipped with some of these intelligent technologies to assist the driver when conditions become difficult to drive in, giving more control and decision making responsibilities to the vehicle. These technologies are added to our vehicles not only to make them more desirable and more enjoyable to drive but most importantly to make them safer by making them more capable. Safety systems have been highly developed over the years and most have gone from being available as optional extras to becoming standard safety equipment, Anti-locking Braking Systems (ABS) and Traction control are two that can now be found as standard systems on modern vehicles. With the advancement and affordability of technology, systems are being introduced that are a lot more intelligent which offer assistance to the driver in the case of difficult driving conditions such as bad weather and/or off-road terrain. This section will discuss some of the intelligent systems available on vehicles today.

\subsection{BMW - iDrive}

With iDrive, BMW has opened up a new development in driver orientation and vehicle control [1]. Certain top of the range BMW vehicles come equipped with the iDrive, which is now in its $3^{\text {rd }}$ generation. iDrive is an onboard computer that allows the user to control the vehicles features using the control knob. Some features can be seen as a luxury such as climate control and satellite navigation; however most features are driver assistive such as the suspension settings as well as the traction control, but the most impressive advanced assistive technology on the iDrive menu has to be the night vision mode that uses a front facing infrared camera to show up any potential obstacles in the dark on the iDrive's LCD screen (Figure 1). This assists the driver in avoiding any obstacles or to see pedestrians that could potentially be unseen in the dark. 


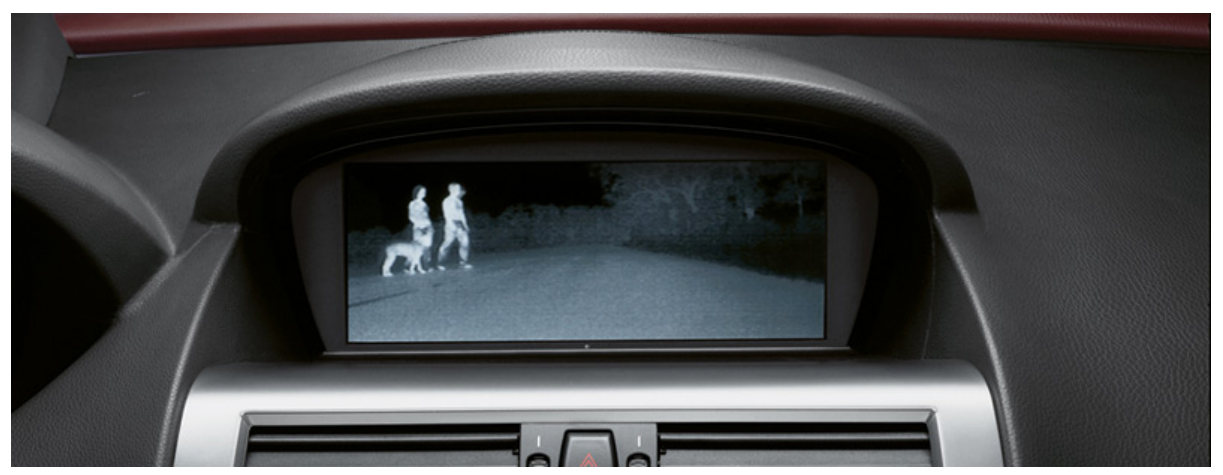

Fig. 1. BMW iDrive's night vision (BMW, 2008)

\subsection{Land Rover - Terrain Response}

Since its release in 2006, The Land Rover Discovery 3 comes complete with an assistive system known as Terrain Response, which allows the driver to select the type of configuration, via an in car dial (Figure 2), in reference to the terrain type that they are about to travel over. The dial has five settings: a general mode, for everyday driving; grass, gravel and snow driving; one for travelling through mud and ruts; sand; and finally a rock crawling mode. For each setting the system will adjust the vehicles characteristics to best suit the terrain type. ABS, traction control, stability control, suspension, the shift schedule of the transmission, the 4WD differentials and even the engines throttle response are all but some of the settings adjusted by the system [2].

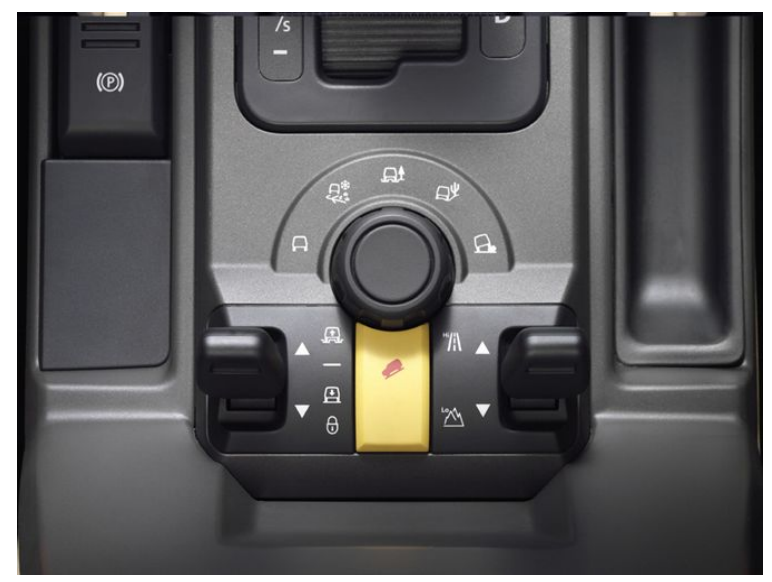

Fig. 2. Terrain Response available on the Land Rover Discovery 3 (Land Rover, 2006)

\subsection{Citroen - Snow Motion}

The 2009 Citroen C5 comes equipped with an assistive system known as Snow Motion. Citroen, who collaborated with Bosch on this system, say it eliminates the 
apprehension of non informed drivers [3]. Snow motion, unlike other anti-skid systems doesn't stop the traction of the wheels but instead assists them independently in order to keep the vehicle driving through bad conditions. It works by analysing the situation using information from the vehicles acceleration, angle of the wheels, slope angle, grip and condition of the road; and if conditions are detected to be adverse then the computer will firstly authorise alternative spinning of the driving wheels to handle the condition of the road, and then the system works to adjust the drive settings by measuring the conditions, evaluating the action of the driver and also the effects of the computer's instructions to the car; aiming to bring together what the driver wants to do with the reality of the road conditions.

\subsection{Nissan - All Mode 4x4-i}

Nissan are another vehicle manufacturer that offers superiority when driving in adverse and off-road conditions. Their solution is the All Mode 4x4-i available on the latest X-Trail, which is a fully integrated system highly publicised to be able to analyse the terrain as you drive [4]. The systems uses information from its network of sensors to make adjustments to the torque distribution to the wheels, 4 wheel drive and ABS settings. There are 4 main modes - uphill start, downhill drive support, automatic torque distribution and finally the Electronic Stability Program (ESP) which works to match the vehicles response to the input at the steering wheel. Nissan believe that the correct adjustments to the settings mentioned previously can assist the driver and make the vehicle highly capable to successfully drive in any condition such as snow, water, rock and sand; whether it is on or off the road.

\section{Terrain Sensing}

The systems discussed in the previous section highlight the need for vehicles to be more intelligent to become more aware of their environment. They need the intelligence to be able to make the correct adjustments to the drive settings; however they use information from internal sensors to work out the external conditions and lack the ability to directly sense the environment. There is therefore a need to directly sense the terrain and its conditions. A system with this ability could eliminate the need to guess the external conditions and make the system more aware of the real-time terrain conditions. This system could also automatically adjust the drive settings to best suit the conditions without having to worry the unaware driver or in the case of the autonomous UGV give it the added awareness that a human driver would have.

To directly sense the terrain the actual interaction at the wheel would need to be measured, which can be done either actively or passively. To actively do this the contact of the tyre to the terrain would need to be measured which would require some type of contact sensor [5], however to do it passively the system must detect the passive result of the interaction; if contact is an active result of the interaction, then noise can be identified as a passive result.

\subsection{Tyre Noise}

As mentioned tyre noise is the passive result of the interaction between tyre and terrain, which will change if either of the two elements in the interaction are changed. If 


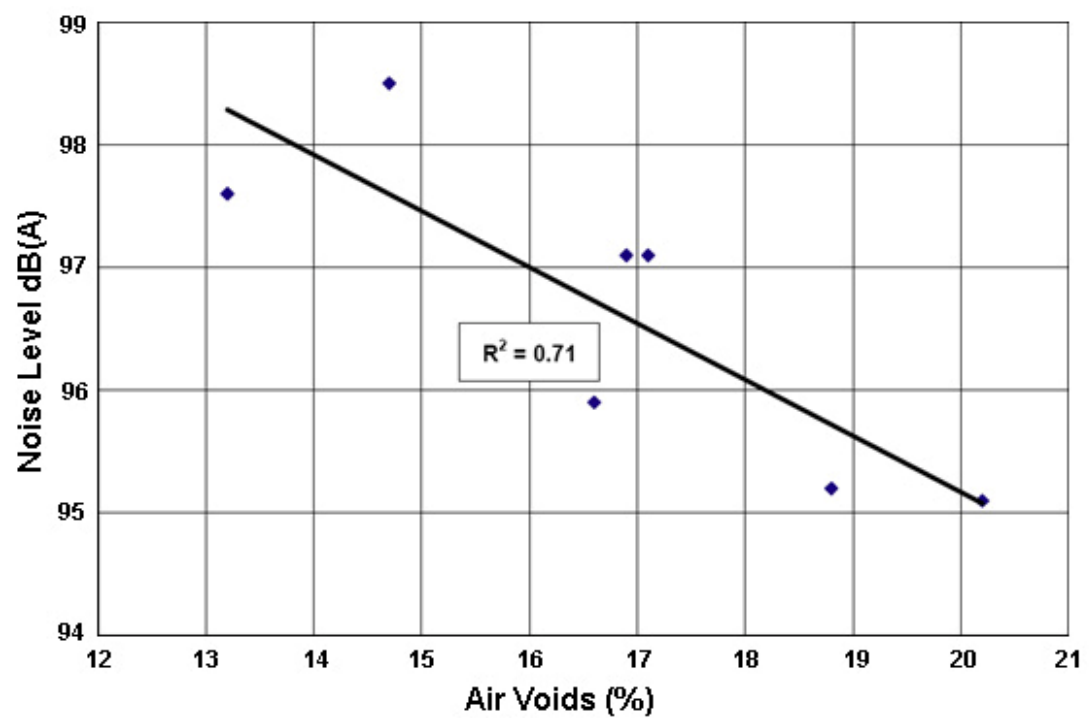

Fig. 3. Graph showing the change in tyre noise levels with a change in air voids (NCAT, 2004)

different tyres will result in different sounds over the same terrain, that also means that the same type of tyre will make different sounds over different terrain and if that difference can be measured then potentially the terrain type can be identified.

The noise which is radiated from the tyre surface is produced by several mechanisms, including the vibration of the tyre surface, vibrations of the tread blocks and resonances of the air cavities in the contact patch between the tyre and the road surface [6] and these can all help to detect the terrain type. The sound caused by the deformation of the tread will give a distinctive sound that can help determine the hardness of the surface; noise from tyre vibrations will help determine the roughness of the surface; the sound of the air passing through the tyre cavities changes when closed which can help determine if the surface is covered, possibly by water (see Figure 3). Other sounds made by the tyre-terrain interaction which could help determine the terrain type are stick-slip and stick-slap sounds which can help to differentiate the density of surface [7].

To be able to sense all the different sounds and changes in them an advanced acoustic sensor (microphone) will need to be used. Further work will be done in testing tyre noise in order to find distinct differences in sound over different terrain conditions.

\section{Conclusion}

In most commercial vehicles, especially those designed to drive off-road or in adverse conditions, come equipped with intelligent systems as discussed earlier. The development and availability of these systems is increasing with the affordability of technology and the increased need for more capable vehicles. The existence of these 
systems highlights a number of points; the first is the need for vehicles to be more intelligent, which they need to be so that they are more aware of their environment in order to cope with changes in terrain conditions. Once the vehicle has the information about its situation then it selects the correct drive configuration highlighting the next point that different terrain types and weather conditions require different drive styles and settings, and therefore vehicles must have adjustability in order to be adaptable to the situation leading to a more capable system.

These intelligent systems are being offered on manned vehicles, therefore systems developed for autonomous UGV's need to be highly intelligent as they don't have the decision making of a human operator and have sole responsibility to select the correct settings to be able to successfully cope with the situation. This shows that an autonomous system needs more information and intelligence to be able to understand its environment, and therefore the sensing of terrain conditions will offer the system more situational awareness about the actual environmental conditions making a more knowledgeable and terrain capable system.

Terrain sensing isn't limited to UGV's as mentioned earlier; it can also be assistive to the current systems, providing more information to help understand what the actual terrain conditions are, and also to make the driver more aware of the external conditions.

\section{References}

1. Quain, J.R.: For iDrive 4.0, BMW Brings Back a Few Buttons. New York times (2008)

2. Vanderwerp, D.: What Does Terrain Response Do? (2005), http: / /www. caranddriver.com/features/9026/ what-does-terrain- response-do.html

3. Citroen. Technology - Snow Motion (2009), http: / /www.citroen.co.uk/technology/safety/snow-motion/

4. Nissan. New X-Trail ALL MODE - Nissan's new 4x4 technology (2007), http: / / www. theallnewxtrail.info/mumfords/allmode4x4/

5. Odedra, S., Prior, S.D., Karamanoglu, M.: Improving the Mobility Performance of Autonomous Unmanned Ground Vehicles by Adding the Ability to 'Sense/Feel' their Local Environment. In: Human Computer Interaction International 2007, Beijing International Convention Center (2007)

6. University of Cambridge, Tyre / road interaction noise - Road surfaces available for experimentation, Department of Engineering (2006)

7. Hanson, D.I., James, R.S., NeSmith, C.: TIire/Pavement Noise Study, National Center for Asphalt Technology. Auburn University, Alabama (2004) 